\title{
Diffusion Processes on Power-Law Small-World Networks
}

\author{
Balázs Kozma, ${ }^{1}$ Matthew B. Hastings, ${ }^{2}$ and G. Korniss ${ }^{1}$ \\ ${ }^{1}$ Department of Physics, Applied Physics, and Astronomy, \\ Rensselaer Polytechnic Institute, $1108^{\text {th }}$ Street, Troy, NY 12180-3590, USA \\ ${ }^{2}$ Center for Non-linear Studies and Theoretical Division, \\ Los Alamos National Laboratory, Los Alamos, New Mexico 87545, USA
}

(Dated: November 5, 2018)

\begin{abstract}
We consider diffusion processes on power-law small-world networks in different dimensions. In one dimension, we find a rich phase diagram, with different transient and recurrent phases, including a critical line with continuously varying exponents. The results were obtained using self-consistent perturbation theory and can also be understood in terms of a scaling theory, which provides a general framework for understanding processes on small-world networks with different distributions of long-range links.
\end{abstract}

PACS numbers: 89.75.Hc, 68.35.Ct, 05.40.Fb, 89.20.Ff

Studying diffusion-related processes on networks, one can gain insight into synchronization [1] and spreading phenomena in natural, artificial, and social systems [2]. In this Letter, we consider a class of models on smallworld (SW) networks [3] with a power-law probability distribution of long-range links, where the probability of two nodes being connected goes as $r^{-\alpha}$, with some exponent $\alpha[4,5,6,6]$. Such a structure can emerge in synchronization problems of distributed computing 8] where synchronization is achieved by introducing random communications between distant processors. Choosing a power-law SW network may be preferable, as it lowers the cost associated with communications. It was also recently argued []] that "wiring-cost" considerations for spatially embedded networks, such as cortical networks [9] or on-chip logic networks [10], can generate such power-law-suppressed link-length distribution. A physical example of such a power-law SW network arises in diffusion on a randomly folded polymer discussed below [1, 12].

The addition of random long-range links to a regular $d$-dimensional network, producing a SW network, leads in many cases to a crossover to mean-field-like behavior [13], effectively becoming equivalent to averaging over the long-range links in an annealed fashion. Here we focus on systems where it is not the case, and the contrast between the quenched and annealed systems is strong [5, 14]. We develop a perturbative method for the quenched network, which is asymptotically exact, as confirmed by numerics.

Varying the exponent $\alpha$, controlling the distribution of length of the links, the diffusive dynamics gives rise to a rich phase diagram, as the connection topology interpolates between the original "plain" SW $(\alpha=0)$ and the purely short-range connected $(\alpha=\infty)$ network. The phase diagram can also be understood in terms of simple scaling ideas, showing that the breakdown of mean-field theory is associated with the relevance of the operator for scattering off a single link.

Diffusion-Related Processes - We start with a $d$ - dimensional lattice of linear size $L$ and add long-range links, connecting two sites with a probability proportional to $p r^{-\alpha}$, where $r$ is the distance between the two sites, $p$ is the probability of a site to have a random link, and $\alpha$ is the exponent of the decay, ranging from 0 to $\infty$. Although the original problem can have only one random link between two distant sites, the analytics are slightly simpler if pairs of sites may be connected with multiple links with a Poisson distribution of links; both cases have the same universal behavior in the small- $p$ limit. Thus, in this work, we set the probability, that sites $i$ and $j$ are connected by $n$ random links, equal to $\frac{\left(p /\left(\mathcal{N}|i-j|^{\alpha}\right)\right)^{n}}{n !} e^{-p /\left(\mathcal{N}|i-j|^{\alpha}\right)}$, where $\mathcal{N}=\sum_{j \neq 0}|j|^{-\alpha}$.

One case in which the diffusion equation arises is a macromolecule randomly moving along a polymer chain jumping over adjacent segments with nonzero probability 11]; if the macromolecule motion is fast compared to rearrangements of the links, then the network may be considered as quenched 11, 15], while if the macromolecule motion is slow compared to the link rearrangements then the network is annealed [11, 12. The equation describing random walk processes is

$$
\partial_{t} P_{\mathbf{r}^{\prime}}(t)=-\sum_{\mathbf{r}^{\prime \prime}}\left(\Delta_{\mathbf{r}^{\prime} \mathbf{r}^{\prime \prime}}+q \Delta_{\mathbf{r}^{\prime} \mathbf{r}^{\prime \prime}}^{r n d}\right) P_{\mathbf{r}^{\prime \prime}}(t)
$$

where $P_{\mathbf{r}^{\prime}}(t)$ is the probability of finding the walker at site $\mathbf{r}^{\prime}$ at time $t,-\Delta_{\mathbf{r}^{\prime} \mathbf{r}^{\prime \prime}}$ is the discretized version of the $d$-dimensional diffusion (or Laplace) operator, and $-\Delta_{\mathbf{r}^{\prime} \mathbf{r}^{\prime \prime}}^{r n d}$ is the diffusion operator on the random links with diffusion coefficient $q$ i.e. in one dimension $\Delta_{i j}=$ $2 \delta_{i, j}-\delta_{i, j-1}-\delta_{i, j+1}$ and $\Delta_{i j}^{r n d}=\delta_{i j} \sum_{l \neq i} J_{i l}-J_{i j}$, where $J_{i j}=n$ if there are $n$ links connecting sites $i$ and $j$. The Green's function, $G_{\mathbf{r}^{\prime}, \mathbf{r}^{\prime \prime}}(t)$, of this model is the solution of the equation with initial condition $P_{\mathbf{r}^{\prime}}(t=0)=\delta_{\mathbf{r}^{\prime}, \mathbf{r}^{\prime \prime}}$. Because the net probability has to be conserved during the process, the transition matrix has a zero eigenmode so the matrix is invertible only in the subspace orthogonal to this mode. Consequently, the limiting case of the temporally Fourier-transformed Green's function, 
$G_{\mathbf{r}^{\prime}, \mathbf{r}^{\prime \prime}}=\lim _{\omega \rightarrow 0}\left(i \omega+\Delta+q \Delta^{r n d}\right)_{\mathbf{r}^{\prime}, \mathbf{r}^{\prime \prime}}^{-1}$, is only invertible in the same subspace. $G_{\mathbf{r}^{\prime}, \mathbf{r}^{\prime}}$, in the context of the random walk, is related to the return probability and its scaling behavior with $L$ will determine whether the walk is transient or recurrent. Earlier numerical work [5] for $d=1$ suggested $\alpha \approx 2$ as a crossover point from the transient to the recurrent phase. Here we compute the scaling properties of the propagator analytically (and confirm it numerically), and precisely construct the full phase diagram for all $d$.

The second case, relevant to synchronization problems on SW networks in a noisy environment, is the EdwardsWilkinson process [14]

$$
\partial_{t} h_{\mathbf{r}^{\prime}}(t)=-\sum_{\mathbf{r}^{\prime \prime}}\left(\Delta_{\mathbf{r}^{\prime} \mathbf{r}^{\prime \prime}}+q \Delta_{\mathbf{r}^{\prime} \mathbf{r}^{\prime \prime}}^{r n d}\right) h_{\mathbf{r}^{\prime \prime}}(t)+\eta_{\mathbf{r}^{\prime}}(t),
$$

where $h$ is the surface height and $\eta$ is a delta-correlated Gaussian white noise with variance 2. In this case, the Green's function is the steady-state two-point correlation function between $h_{\mathbf{r}^{\prime}}$ and $h_{\mathbf{r}^{\prime \prime}}$, so $G_{\mathbf{r}^{\prime}, \mathbf{r}^{\prime \prime}}=\left\langle h_{\mathbf{r}^{\prime}} h_{\mathbf{r}^{\prime \prime}}\right\rangle=$ $\left(\Delta+q \Delta^{r n d}\right)_{\mathbf{r}^{\prime}, \mathbf{r}^{\prime \prime}}^{-1}$, and again, the function is defined in the space orthogonal to the uniform zero mode. In the context of the Edwards-Wilkinson equation, $G_{\mathbf{r}^{\prime}, \mathbf{r}^{\prime}}$ is the measure of the surface roughness or width [14].

Ultimately, we are interested in the average of these quantities over all realizations of the networks. This averaging (denoted by [ ]) restores translation invariance for $\left[G_{\mathbf{r}^{\prime}, \mathbf{r}^{\prime \prime}}\right]$ and therefore its spatial behavior will depend only on the difference $\mathbf{r}^{\prime \prime}-\mathbf{r}^{\prime}$. Hence, we define

$$
G(\mathbf{r}) \equiv\left[\left(\Delta+q \Delta^{r n d}\right)^{-1}\right]_{\mathbf{r}^{\prime}, \mathbf{r}^{\prime}+\mathbf{r}},
$$

where $\mathbf{r}$ is the spatial separation of the two sites. We expect that when the probability of the random links decays rapidly enough $(\alpha \rightarrow \infty)$, the large scale behavior of the system is not affected by these links. When the probability of the random links decays slowly $(\alpha \rightarrow 0)$, the results of the original SW network should be recovered 14. In between, there is a transition which is the focus of this Letter. Our starting point is an annealed calculation that turns out to be the first order result of a naive perturbation expansion for the quenched system. For small $p$ this expansion breaks down and we apply a self-consistent calculation.

Annealed Calculation - First, we consider the annealed predictions. In this calculation, to approximate the behavior of $G(\mathbf{r})$, the random links are replaced with their expected number, i.e. the random interactions are annealed [5, 11, 12, 16]. That is, in (3), $\Delta^{r n d}$ is substituted with $\left[\Delta^{r n d}\right] . \quad\left[\Delta^{r n d}\right]_{\mathbf{r}, \mathbf{r}}=p$, and $\left[\Delta^{r n d}\right]_{\mathbf{r}, \mathbf{r}^{\prime}}=\frac{-p}{\mathcal{N}} \frac{1}{\left|\mathbf{r}-\mathbf{r}^{\prime}\right|^{\alpha}}$ for $\mathbf{r} \neq \mathbf{r}^{\prime}$. Because of its translational invariance, this operator is diagonal in Fourierspace, likewise $\Delta$. In the large system-size limit, its eigenvalues in Fourier basis are: $\left[\Delta^{r n d}\right](\mathbf{k}) \propto p$ for $0<\alpha<d ;\left[\Delta^{r n d}\right](\mathbf{k}) \propto p k^{\alpha-d}$ for $d<\alpha<d+2$;

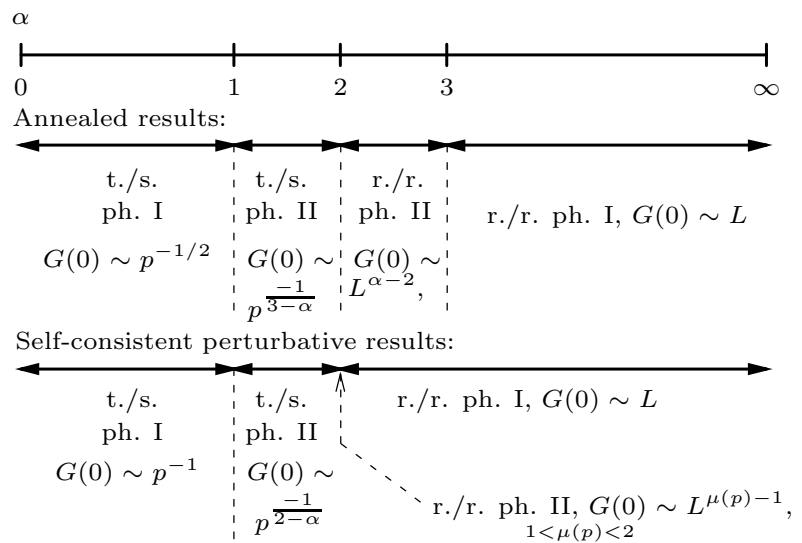

FIG. 1: Phase diagram for one dimension. Results from the annealed calculation (top) and from the self-consistent perturbative calculation for the quenched case (bottom) showing different behavior in the transient/smooth phases (t./s. ph.) and in the recurrent/rough (r./r.) ones.

and $\left[\Delta^{r n d}\right](\mathbf{k}) \propto p k^{2}+\ldots$ for $d+2<\alpha$. Hence, $G(\mathbf{r})$ can be obtained by inverse Fourier-transformation and

$$
G(\mathbf{0})=\frac{1}{L^{d}} \sum_{\mathbf{k} \neq 0} \frac{1}{k^{2}+q\left[\Delta^{r n d}\right](k)}
$$

To calculate the scaling properties of this quantity, with respect to $p, q$, and $L$, the sum can be approximated with its integral-form limit. The results for one dimension are sketched in Fig. 11 (top). For $\alpha<2$, the integral (4) converges at both ends, so is system size independent for large $L$ and diverges as $p \rightarrow 0$. There are two regimes, distinguished by their scaling behavior (reflecting the properties of the underlying random walk/surface) : (1) transient/smooth phase $I$, where the system behaves as if the long range links were uniformly distributed, and $G(0)$ is $\alpha$-independent; (2) transient/smooth phase II, where $G(0)$ is still finite in the thermodynamic limit, but has an $\alpha$-dependent divergence as $p \rightarrow 0$. For $\alpha>2, G(0)$ has an infrared divergence resulting in two system-size dependent regimes: (1) recurrent/rough phase $I$, where the system behaves as if there were no long range links, and $G(0)$ diverges linearly with the system-size, $L ;(2)$ recurrent/rough phase II, with sublinear scaling with respect to the system-size. At the boundaries of these phases, logarithmic corrections are present.

Self-consistent Perturbative Calculation for the Quenched Network - Following [14], we calculate $G(\mathbf{0})$ perturbatively, treating $\Delta^{r n d}$ as a weak scattering potential. Expanding Eq.(3), $G=$ $G^{0}-G^{0}\left[q \Delta^{r n d}\right] G^{0}+G^{0}\left[q \Delta^{r n d} G^{0} q \Delta^{r n d}\right] G^{0}-\ldots$, where $G^{0}=\Delta^{-1}$, the Green's function with no random interactions introduced. Rearranging the formula, one obtains the Dyson equation, $G=G^{0}-G^{0} \Sigma G=(\Delta+\Sigma)^{-1}$, where $\Sigma=q\left[\Delta^{r n d}\right]-q^{2}\left[\Delta^{r n d} G \Delta^{r n d}\right]_{c}+\ldots$ is the self-energy and [ $]_{c}$ represents the cumulants of the random variables $\Delta_{\mathbf{r}, \mathbf{r}^{\prime}}^{r n d}$, for example 
$\left[\Delta_{\mathrm{ij}}^{r n d} \Delta_{\mathrm{kl}}^{r n d}\right]_{c}=\left[\Delta_{\mathrm{ij}}^{r n d} \Delta_{\mathrm{kl}}^{r n d}\right]-\left[\Delta_{\mathbf{i j}}^{r n d}\right]\left[\Delta_{\mathbf{k l}}^{r n d}\right]$ and so on. Solving the equation to first order, $\Sigma=q\left[\Delta^{r n d}\right]$, the annealed result is recovered. Substituting this first-order result into the next order term of $\Sigma,-q^{2}\left[\Delta^{r n d} G \Delta^{r n d}\right]_{c}$, it can be shown that the correction becomes dominant over the first-order term as $p \rightarrow 0$, and so the perturbation expansion breaks down.

To have an operative perturbation expansion, as a first step $\Sigma$ has to be calculated involving all the scatterings from a single link, then corrections representing scatterings from multiple links may be successively obtained. The closed form of $\Sigma$ for the single-link problem is

$$
\Sigma^{s l}(\mathbf{r} \neq \mathbf{0})=-q \frac{p}{\mathcal{N} r^{\alpha}} \frac{1}{1+2 q(G(\mathbf{0})-G(\mathbf{r}))}
$$

and $\Sigma^{s l}(\mathbf{0})=-\sum_{\mathbf{r} \neq \mathbf{0}} \Sigma^{s l}(\mathbf{r})$. Since $G$ and $\Sigma^{s l}$ are coupled, they have to be solved for self-consistently. To do this, we make an ansatz for $\Sigma^{s l}$, namely, in Fourier-space, $\Sigma^{s l}(k)=s k^{\mu}$ for small $k$-s, $s$ and $\mu$ to be determined. Using this form of $\Sigma^{s l}$, the Green's function, in Fourier space, becomes $G(k)=\left(k^{2}+s k^{\mu}\right)^{-1}$. Its long-distance real-space behavior, as in the annealed case, can be calculated with the integral-form limit of the expressions and $\Sigma^{s l}$ should be treated similarly.

For one dimension, as $p \rightarrow 0$ at non-vanishing $q$, one finds several cases. For $\alpha<1, \mu=0, s=p^{2}, G(0) \propto p^{-1}$. For $1<\alpha<2, \mu=\alpha-1, s=p^{\frac{3-\alpha}{2-\alpha}}, G(0) \propto p^{\frac{-1}{2-\alpha}}$. For $\alpha>2, \mu=\alpha, s=p, G(0) \propto L$, while for $\alpha=2, \mu$ is given by the solution of the self-consistent formula: $p \frac{3}{2 \pi^{2}} \frac{\cos \left(\frac{\mu \pi}{2}\right) \Gamma(-\mu)}{\sin \left(\frac{\mu \pi}{2}\right) \Gamma(1-\mu)}=1,1<\mu<2$. The leading order results are sketched in Fig. 1 (bottom). Similar phases appear as were predicted by the annealed argument, but in transient/smooth phase II, we have a different scaling property, while recurrent/rough phase I spans a wider interval of the $\alpha$-axis. Finally, recurrent/rough phase II is collapsed to one point on this axis, and in this phase, the sublinear behavior of $G(0)$ is no longer determined by the distance-distribution of the random links but rather by their density, $p$, through the self-consistent formula. The form of this equation is approximate, but the fact that the exponent $\mu$ depends continuously on $p$ is likely to be correct, in light of the scaling arguments below.

Scaling - These results for the quenched network can be understood via a simple scaling argument. The Green's function has dimension $l^{2-d}$, where $l$ is some length scale, as can be seen by considering the Green's function $G^{0}(r)=\int \mathrm{d}^{d} k \exp (i k r) / k^{2}$. The dimension of $q$ is $l^{d-2}$, as $q G$ is dimensionless.

The dimension of $p$ is $l^{-d}$ for $\alpha \leq d$ and $l^{\alpha-2 d}$ for $\alpha \geq d$. This arises from perturbation theory, but can also be understood geometrically: the probability that a block of linear size $l$ has a long-range link with a length at least $l$ is $p l^{d}$ for $\alpha \leq d$ and $p l^{2 d-\alpha}$ for $\alpha \geq d$. Thus, at a length scale $l$, for $\alpha \geq d$, the important dimensionless quantities are: $\tilde{q}=q l^{2-d}, \tilde{p}=p l^{2 d-\alpha}$, and $\tilde{\omega}=\omega l^{2}$.

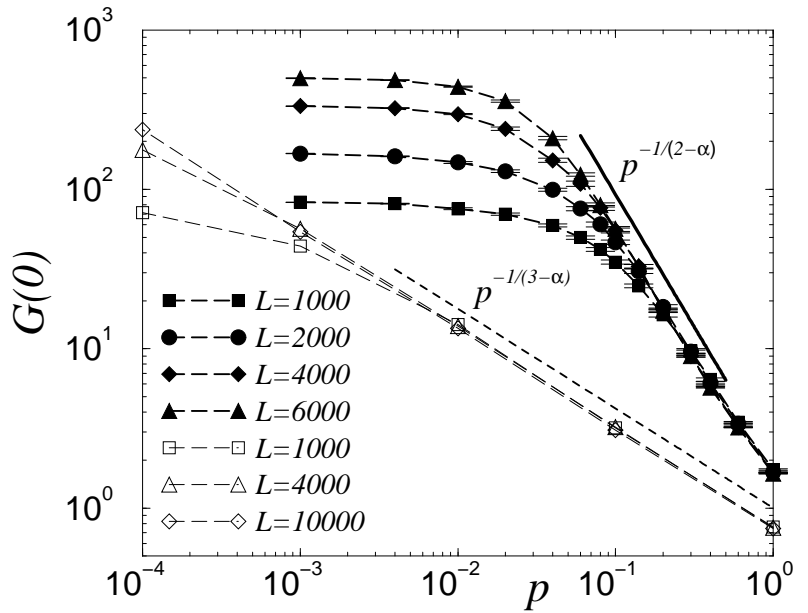

FIG. 2: Finite-size propagators $G(0)$ vs. $p$ for $\alpha=1.4, q=1$ (transient/smooth phase II) for various system sizes. Solid symbols represent data obtained by exact numerical diagonalization averaged over 100 realizations for the quenched network. The solid straight line represents the slope obtained from the perturbative analytic calculations in the $L \rightarrow \infty$ limit. Open symbols correspond to the steady-state averaged propagator on the annealed network with the straight dashed line being the mean-field analytic result in the $L \rightarrow \infty$ limit.

There are several cases in the limit of small $p$ and nonvanishing $q$. If $2 d<\alpha$, then $\tilde{p}$ scales to zero, and there are no links at large length scales; the system looks like a network without long-range links, giving $G(0) \propto L$ as in recurrent/rough phase I.

If instead $2 d>\alpha>d$, then at some scale $l \propto$ $p^{-1 /(2 d-\alpha)}$, the density of long-range links becomes of order unity. This case breaks into two further subcases. If $d<2$, then $q$ is relevant; if $p$ is small and $q$ is nonvanishing, then higher order corrections will cut off the growth in $\tilde{q}$, so $\tilde{q}$ at scale $l$ will be of order unity, and will be independent of $q$ at lattice scale. This tells us that the only meaningful dimensional number is $p$ (since $q$ is not important at this scale), and dimensional analysis gives $G(0) \propto p^{-(2-d) /(2 d-\alpha)}$ as in transient/smooth phase II. This is a case in which mean-field theory breaks down because there is typically only one link at the scale $l \propto p^{-1 /(2 d-\alpha)}$ and the scattering off links is strong. If instead $d>2$, then $\tilde{q}$ decreases with increasing $l$, and at $l \propto p^{-1 /(2 d-\alpha)}$ the system has a high density of weak links where mean-field theory is valid. Near $d=2$, an $\epsilon$ expansion is available 17 to compute higher corrections and determine prefactors in $G(0)$. Finally, for $\alpha=2 d, \tilde{p}$ is marginal, giving the continuously varying exponent of recurrent/rough phase II.

In the annealed case, one has a single number $p q$, and the corresponding dimensionless coupling constant is $\tilde{p q}=p q l^{d+2-\alpha}$. This becomes relevant for $\alpha<d+2$, and, e.g., yields the scaling $G(0) \propto(p q)^{-(2-d) /(d+2-\alpha)}$ in the transient/smooth phase II.

Numerical Results - For the annealed case, we numer- 


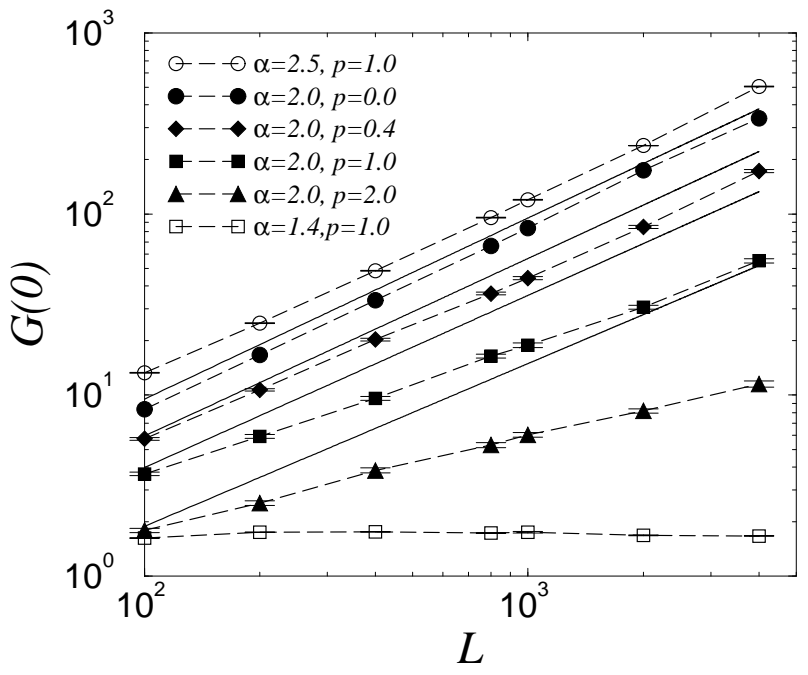

FIG. 3: $\quad G(0)$ vs the system size $L$ (all data for $q=1$ ) from exact numerical diagonalization. Data in the recurrent/rough phase I ( $O$ symbols) indicate the linear scaling with $L$ (data is shifted up for clarity). Filled symbols represent the scaling behavior in the recurrent/rough phase II $(\alpha=2)$ for various values of $p$, indicating the $p$-dependence of the exponent. The solid lines, representing the slopes obtained from the selfconsistent formula for the same set of values of $p$, show significant deviations from the corresponding data for larger values of $p$. Data in the transient/smooth phase II ( $\square$ symbols), indicating a finite $G(0)$ in the $L \rightarrow \infty$ limit, are also shown for comparison.

ically integrated Eq. (2) where the underlying power-law SW network was also reconfigured at every timestep, and measured the width $G(0)$ in the steady state. The results in Fig. 2] indicate that the mean-field approximation is exact for the annealed system. For the quenched case, we compared our analytic results to those obtained from exact numerical diagonalization of the discretized Laplacian for representative points in the phase diagram. In Fig. 2 we show the main features of transient/smooth phase II $(\alpha=1.4)$. For a region of $p$ values, good agreement between the two results can be observed. For finite systems of size $L$, the crossover in $G(0)$ (see below) occurs when $p<p_{\times} \propto L^{\alpha-2}$. For large values of $p$, deviation can be observed due to possible higher order corrections in $p$. In Fig. 3 we show the $L$-dependence of $G(0)$ in the recurrent/rough phases. While in the recurrent/rough phase I it diverges as $G(0) \propto L$, in the somewhat "degenerate" recurrent/rough phase II (collapsed onto a single point, $\alpha=2)$ the exponent of the divergence depends continuously on $p, G(0) \propto L^{\mu(p)-1}$. Although the self-consistent formula (solved numerically) predicts this latter feature qualitatively, the actual exponent appears to be affected by higher order corrections.

Crossovers - There are two competing terms determining the large scale behavior of the Green's function, the diffusion through the regular links, $\Delta$, and through the random ones, $\Delta^{r n d}$. For small system sizes, the num- ber of the random links is small, their contribution to the ensemble average is negligible, the behavior is close to that of a $d$-dimensional unperturbed system. The effect of the random interactions takes over when the selfenergy generated by these random links start to dominate the infrared behavior of $G(k)$, which is when $k_{\times}^{2} \approx \Sigma\left(k_{\times}\right)$. Thus, for the quenched case, in the transient/smooth phase II in one dimension, for $L \ll L_{\times}=k_{\times}^{-1} \propto p^{\frac{-1}{2-\alpha}}$, $G(0) \propto L$; for $L \gg L_{\times}, G(0) \propto p^{\frac{-1}{2-\alpha}}$. For the annealed system, the corresponding crossover occurs at $L_{\times} \propto p^{\frac{-1}{3-\alpha}}$, leading to $G(0) \propto p^{\frac{-1}{3-\alpha}}$ for $L \gg L_{\times}$.

Discussion - We investigated diffusion related processes on SW networks with distance dependent distribution of the random links. The results are summarized in the phase diagram of Fig. 1, with dramatically different behavior between the quenched and annealed case. The results for the quenched network can be understood via simple scaling arguments, based on the probability of finding links at a given length scale. We expect that these scaling arguments can be generalized to systems with an arbitrary probability $p(r)$ of having long-range links and thus will be applicable to a wide-range of networks in which mean-field theory breaks down.

Acknowledgments - This work was supported by NSF Grants DMR-0113049, DMR-0426488, the Research Corporation, and by US DOE contract W-7405-ENG-36. BK also acknowledges support from the LANL Summer Student Program.

[1] A. E. Motter, C. Zhou, and J. Kurths, Phys. Rev. E 71, 016116 (2005).

[2] M.E.J. Newman, Social Networks 27, 39 (2005).

[3] D. J. Watts and S. H. Strogatz, Nature (London) 393, 440 (1998).

[4] J. M. Kleinberg, Nature 406, 845 (2000)

[5] S. Jespersen and A. Blumen, Phys. Rev. E 62, 6270 (2000).

[6] C. F. Moukarzel and M. Argollo de Menezes, Phys. Rev. E 65, 056709 (2002)

[7] T. Petermann and P. De Los Rios, arXiv:cond-mat/0501420 (2005).

[8] G. Korniss, M. A. Novotny, H. Guclu, Z. Toroczkai, and P. A. Rikvold, Science 299, 677 (2003).

[9] S. B. Laughlin and T. J. Sejnowski, Science 301, 1870 (2003).

[10] J. A. Davis, V. K. De, and J. D. Meindl, IEEE Trans. Elec. Dev. 45, 580 (1998).

[11] I. M. Sokolov, J. Mai, and A. Blumen, Phys. Rev. Lett. 79, 857 (1997).

[12] D. Brockmann and T. Geisel, Phys. Rev. Lett. 91, 048303 (2003).

[13] M. B. Hastings, Phys. Rev. Lett 91, 098701 (2003).

[14] B. Kozma, M. B. Hastings, and G. Korniss, Phys. Rev. Lett. 92, 108701 (2004).

[15] R. Monasson, Eur. Phys. J. B 12, 555 (1999).

[16] B. Bergersen, Z. Rácz, Phys. Rev. Lett. 673047 (1991) 
[17] M. B. Hastings, Eur. Phys. J. B 42, 297 (2004). 